\title{
Thermal Annealing of Pb Films on Modified Silicon Surface: Electrical Conductance Measurement
}

\author{
Z. KORCZAK* AND T. KWAPIŃSKI \\ Institute of Physics, M. Curie-Skłodowska University, pl. M. Curie-Skłodowskiej 1, PL-20031 Lublin, Poland \\ (Received September 12, 2018; in final form November 21, 2018)

\begin{abstract}
The electron transport properties of thin $\mathrm{Pb}$ films on $\mathrm{Si}(111)-(6 \times 6) \mathrm{Au}$ surface are studied experimentally using the four-point probe method and reflection high-energy electron diffraction system in UHV condition. We have measured the electrical conductance of the substrate with deposited at liquid nitrogen temperature $\mathrm{Pb}$ atoms during annealing and cooling down. We have found that the conductance increases during this process and from comparison of both heating and cooling curves the $\mathrm{Pb}$ recrystallization temperature was determined. Moreover, it was shown that $4 \mathrm{ML}$ thick $\mathrm{Pb}$ film reveals temperature dependence of the conductance corresponding to the metallic bulk structure with the temperature coefficient of resistivity equal to $0.00361 / \mathrm{K}$. To describe theoretically the conductance of thin $\mathrm{Pb}$ films the modified Trivedi-Ashcroft theory with temperature dependence of the roughness function was used in our calculations.
\end{abstract}

DOI: 10.12693/APhysPolA.134.1211

PACS/topics: 73.61.-r, 73.50.--h, 73.25.+i

\section{Introduction}

Electron transport properties of thin atomic films have attracted significant theoretical and experimental interest for many years mainly due to their potential applications in nanoelectronics, e.g. [1]. Spatial confinement of the electron motion in the direction normal to the surface leads to quantization of the momentum which is responsible for many effects like the Friedel oscillations [2, 3], quantum-size effect (QSE) and oscillations of the electrical resistivity $[4,5]$, the Hall effects [6-8], to name a few. Recently, also non-trivial topological properties of two-dimensional systems were investigated in many $2 \mathrm{D}$ compounds e.g. $[9,10]$. These systems, in comparison with bulk materials, exhibit new physics with protected conducting edge states. Thin metallic films are often fabricated on different substrates using epitaxial methods in ultra high vacuum (UHV). The best results have been obtained on $\mathrm{Si}$ or Ge surfaces with well-known superstructures as for them the growth mode of many atoms (e.g. $\mathrm{Pb}, \mathrm{Au}, \mathrm{In}, \mathrm{Ag}$ ) is close to the layer-by-layer growth. Even small number of deposited atoms can drastically change the conductance of the system [11-15]. Movement of atoms on such surfaces can lead to formation of atomic clusters, islands or chains (self-organized phenomena) [16-19]. Many of these processes are governed by atom diffusion at the substrate [20-24].

Especially interesting are temperature measurements of metallic thin-films conductance - electronic properties of such structures can be controlled by the sample temperature and are important in nanoelectronics. The conductance of thin films can suddenly change at a particular temperature which can be related with

*corresponding author; e-mail: zbigniew.korczak@umcs.pl structural or structural-less transitions like e.g. surface recrystallization or metal-isolator transition. The most common method to measure the conductance of thin films is the four-point probe method in the van der Pauw or linear geometry [12, 25-28]. However, the measured conductance signal consists of several channels: though unperturbed bulk crystal, topmost substrate layer, superstructure layer, and thin metallic films. In the literature temperature dependence of the conductance was measured for different surfaces e.g. [12, 29-32] and often the surface recrystallization process together with QSE were observed. However, very little attention has been paid on thermal annealing of ultrathin $\mathrm{Pb}$ films on $\mathrm{Si}(111)$ $6 \times 6$ Au crystal reconstruction. Such a substrate reveals a metallic-like character due to high-density $\mathrm{Au}$ domain walls forming this superstructure and provides regular layer-by-layer $\mathrm{Pb}$ growth mode [6, 29, 33-35]. Then it is rather desirable, even from a practical point of view, to measure temperature characteristics of the conductance for this substrate.

In this paper we analyze the transport properties of ultrathin metallic $\mathrm{Pb}$ films on $\mathrm{Si}(111)-6 \times 6 \mathrm{Au}$ substrate, focusing on the conductance changes during annealing process (heating and cooling of the sample). The aim of our studies is to determine the electrical character of $\mathrm{Si}(111)-6 \times 6 \mathrm{Au}$ surface depending on the $\mathrm{Pb}$ film thickness and to find the surface recrystallization temperature, at which $\mathrm{Pb}$ atoms form regular flat structures (islands) at the substrate. To tackle these problems we analyze the normalized difference between the heating and cooling conductance curves. The conductance measurements are supported by the reflection high-energy electron diffraction (RHEED) experiments which allow us to control the surface morphology. The experimental results of the conductance are supplemented by theoretical calculations within modified Trivedi-Ashcroft theory [36] with temperature-dependence of the roughness 
function. The paper is organized as follows. In Sect. 2 we describe the experimental setup and analyze the results for submonolayer $\mathrm{Pb}$ coverage of the surface and for thin $\mathrm{Pb}$ film. The theoretical formalism based on the Trivedi-Ashcroft theory is presented in Sect. 3. The main conclusions are drawn in the last section of the paper, Sect. 4.

\section{Experimental results and discussions}

The experiments were performed in an ultrahigh vacuum chamber at pressures of the order of $5 \times 10^{-10}$ Torr, equipped with a RHEED apparatus. We have used $n$-type $\operatorname{Si}(111)$ sample with $25 \Omega \mathrm{cm}$ specific resistivity and $18 \times 4 \times 0.4 \mathrm{~mm}^{3}$ size. After polishing, the sample was etched in $1 \mathrm{HF}+10$ ethanol, rinsed in deionized water and mounted on a pair of Mo rods and clamped with Ta foils. Electrical conductivity were measured in situ by the four-point probe method and all electrical contacts were arranged in one line on the substrate. The effective contact area of these macroscopic electrodes does not change during the experiments thus they do not affect the conductance measurements. Very high impedance preamplifier with FET input and SR 830 Lock-in were used in our experiments. An alternating current $I=2 \mu \mathrm{A}, 17 \mathrm{~Hz}$ was applied through the outermost Ta clamps contacts and the $\mathrm{AC}$ voltage was measured across two inner $\mathrm{W}$ wires (1.5 $\mathrm{mm}$ apart) kept in elastic contact with the wafer. The sample holder was mounted to SuperTranVP continuous flow cryostat cold finger (Janis Research Comp. Inc.) and the sample temperature was measured with the AuFe chromel thermocouple being in contact with the wafer. Before measurement run, the surface was cleaned by several flash heatings $(5 \mathrm{~s})$ with a direct current of $13.5 \mathrm{~A}$ through the sample which led to a clear $\operatorname{Si}(111)-7 \times 7$ surface reconstruction. This process was controlled in situ by the RHEED system. In order to prepare $\mathrm{Si}(111)-6 \times 6 \mathrm{Au}$ reconstruction $1.3 \mathrm{ML}$ of $\mathrm{Au}$ was deposited onto the $\mathrm{Si}(111)-7 \times 7$ superstructure at about $950 \mathrm{~K}$ and then the temperature was gradually decreased $(10 \mathrm{~K} / \mathrm{min})$ to room temperature (RT). Next, well ordered sample was cooled down below $90 \mathrm{~K}$ and $\mathrm{Pb}$ atoms were evaporated from Ta crucible at rate $0.02 \mathrm{ML} / \mathrm{min}$ and deposited onto $\mathrm{Si}(111)-6 \times 6 \mathrm{Au}$ substrate. At this temperature $\mathrm{Pb}$ atoms remain almost frozen at the surface [24]. The amount of $\mathrm{Pb}$ in units of monolayer (ML $=7.8 \times 10^{14}$ atoms $/ \mathrm{cm}^{2}$ ) was monitored with a quartz crystal oscillator and RHEED specular beam intensity oscillation during the epitaxial growth has been used for calibration (in our experiment $1 \mathrm{ML}$ of $\mathrm{Pb}$ corresponds to $45.7 \mathrm{~Hz}$ ). Such prepared sample with $\mathrm{Pb}$ film was ready to thermal experiments leading to reorganization (recrystallization) of $\mathrm{Pb}$ atoms at the surface.

RHEED diffraction pictures were used to control the surface crystalline order. However, the detailed morphology of $\mathrm{Pb}$ at $\mathrm{Si}(111)-6 \times 6 \mathrm{Au}$ surface was supported by the scanning tunneling microscopy (STM) topographic images obtained earlier in the literature [33, 35, 37, 38].
The STM measurements indicate that above $180 \mathrm{~K}$ one observes a strongly correlated mesh with regular periodicity and symmetry whereas below this temperature such regularity is not observed [33]. The appearance of $\mathrm{Pb}$ flat terraces or islands during the annealing process was also observed for $\mathrm{Pb}$ films on $\mathrm{Si}(111)-7 \times 7$ [39, 40] or for $\mathrm{Si}(111)-\sqrt{3} \times \sqrt{3}$ reconstruction $[21,41]$. Similarly, $\mathrm{Pb}$ films deposited onto $\mathrm{Ge}(001)$ substrate show the surface recrystallization temperature at about $200 \mathrm{~K}$ [42].

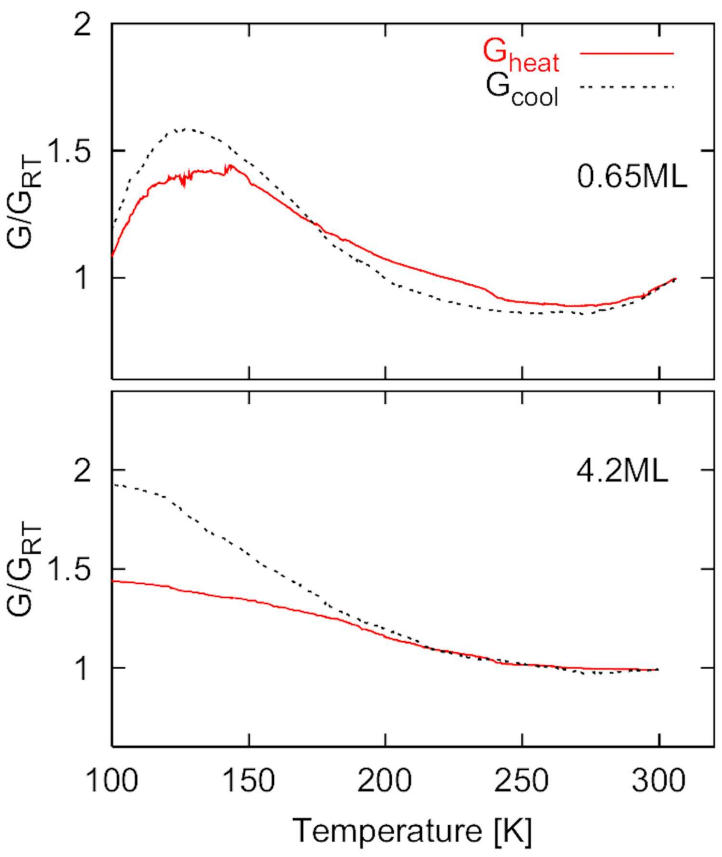

Fig. 1. Temperature dependence of the relative conductance, $G / G_{R T}$, for $0.65 \mathrm{ML}$ of $\mathrm{Pb}$ (upper part) and 4.2 $\mathrm{ML}$ of $\mathrm{Pb}$ (bottom part) deposited on $\mathrm{Si}(111)$ $6 \times 6 \mathrm{Au}$ surface at $90 \mathrm{~K}$. The solid (broken) lines correspond to the conductance during the first heating (cooling) run. $G_{R T}$ stands for the conductance at $300 \mathrm{~K}$.

After deposition of $\mathrm{Pb}$ the temperature dependence of the conductance was measured for many $\mathrm{Pb}$ thicknesses. Annealing and the following cooling were performed very slowly, $1 \mathrm{~K} / \mathrm{min}$ and all data were collected by the computer system. Figure 1 shows the relative conductance, $G / G_{R T}$, as a function of temperature during the first heating run (solid line) and cooling run (broken line) for two chosen thicknesses i.e. 0.65 ML of $\mathrm{Pb}$ (submonolayer coverage) and 4.2 $\mathrm{ML}$ of $\mathrm{Pb}$ (few-monolayer coverage), upper and bottom part, respectively. Here, $G_{R T}$ means the conductance of the system at $300 \mathrm{~K}$ and for the coverage $4.2 \mathrm{ML}$ equals $0.4(\Omega \mathrm{cm})^{-1}$ (in this case the measured voltage drop between $\mathrm{W}$-electrodes is $0.13 \mathrm{mV})$. As one can see, the conductance measured during the first heating and cooling shows the irreversible changes (hysteresis) but all further heating and cooling lead to reversible changes with temperature (they overlap with the broken curves). The conductance of the sample increases after the cycle of heating and cooling due to annealing defect for both, thin and thick $\mathrm{Pb}$ films. During this process 
the surface crystalline order of $\mathrm{Pb}$ is improved. Note that for thin metallic films on anisotropic (e.g. vicinal) surfaces the conductance can decrease after the annealing process [19, 28]. Moreover, for 4.2 ML Pb film (bottom part) the heating and cooling conductance curves converge at about $175 \mathrm{~K}$ and they take almost the same values for higher temperature. It is a consequence of the surface recrystallization of $\mathrm{Pb}$ atoms and formation of even-ML-thick areas (islands) at the surface. Such structure were observed in the STM measurements in our laboratories [35] for somewhat higher temperature (180 K). These $\mathrm{Pb}$ flat areas are relatively large (tens of $\mathrm{nm}$ ), they are very stable and do not change with heating or cooling of the sample between liquid nitrogen temperature (LNT) and room temperature (RT). For submonolayer coverage (upper part in Fig. 1) the conductance curves also converge and cross each other at the temperature equals about $T=172 \mathrm{~K}$, but the curves differ a little for higher-temperature regime. It could be a consequence of high diffusion of $\mathrm{Pb}$ atoms at $\mathrm{Si}$ surfaces (cf. the results of $\mathrm{Pb}$ at $\mathrm{Si}(553)-6 \times 6 \mathrm{Au},[24])$. In such a case $\mathrm{Pb}$ atoms can form small stable atomic clusters with single $\mathrm{Pb}$ atoms moving at the surface between them, even at higher temperatures [33]. The average size of $\mathrm{Pb}$ clusters at this surface can be estimated form the STM measurements [37, 38] and equals about $2 \mathrm{~nm}$ (for submonolayer coverages) whereas the average height of these clusters is about $0.42 \mathrm{~nm}$.

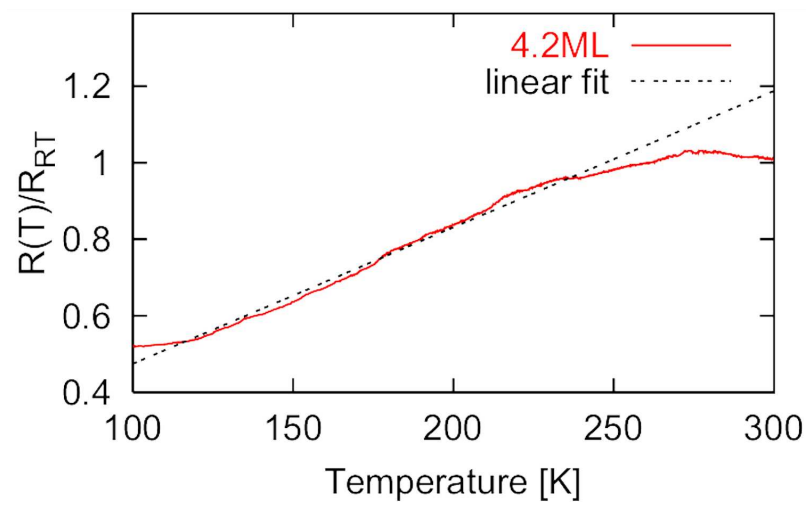

Fig. 2. Temperature dependence of the relative resistance, $R(T) / R_{R T}$, for $4.2 \mathrm{ML}$ of $\mathrm{Pb}$ (solid red line) and corresponding linear fit with the slope coefficient $a=0.0036 \pm 0.5 \% 1 / \mathrm{K}$ (the temperature range of fitting: $100-260 \mathrm{~K})$.

After annealing the conductance changes are reversible and we observe that for thicker $\mathrm{Pb}$ films $(4 \mathrm{ML} \mathrm{Pb})$ they show bulk-like behaviour of $G$. It means that the resistance is proportional to the temperature and linear approximation can be used in this case: $R(T) / R_{R T}=$ $1+\alpha T$, where $\alpha$ is called the temperature coefficient of resistivity (for bulk $\mathrm{Pb}: \quad \alpha=0.00391 / \mathrm{K}[43]$ ). To corroborate this effect we plot in Fig. 2 the relative resistance as a function of temperature and linear fit of this curve for the temperature regime 100-260 K. The obtained temperature coefficient of resistivity in our case equals $0.00361 / \mathrm{K}$, which is in good agreement with the literature data for bulk $\mathrm{Pb}$. Note that for higher temperatures some deviations from the linear values are visible which are related mainly with the semiconductor resistance of the substrate (it decreases with temperature). Moreover, even for metallic bulk materials the resistance depends on many effects (like electron scattering by phonons, electron interactions) and is given by the Bloch-Grüneisen relation which in general is non-linear.

It is worth noting that for ultrathin films the bulk behaviour of the conductance strongly depends on the substrate reconstruction e.g. for $\operatorname{Si}(111)-7 \times 7$ reconstruction it was observed for much more thicker $\mathrm{Pb}$ films, about 10 ML [12], as well as for $\operatorname{Si}(111)-\sqrt{3} \times \sqrt{3}$ reconstruction [32]. Moreover, in our case, the conductance for 4.2 ML film monotonically decreases with increasing temperature but for mentioned above experimental papers (and for the same $\mathrm{Pb}$ coverage) it increases with temperature, showing the surface recrystallization point at about $90 \mathrm{~K}$ (for $4 \mathrm{ML}$ ) or $160 \mathrm{~K}$ (for $6 \mathrm{ML}$ or larger) [12] and at about $165 \mathrm{~K} \mathrm{[32]} \mathrm{(for} 6.1 \mathrm{ML}$ ). It shows a significant advantage of $\mathrm{Si}(111)-6 \times 6 \mathrm{Au}$ surface as one needs only $4 \mathrm{ML} \mathrm{Pb}$ film to ensure bulk properties of the sample and, additionally, the recrystallization temperature is higher in comparison with other Si reconstructions.

The conductance behaviour for submonolayer coverages of $\mathrm{Pb}$ changes with increase and decrease of temperature (even after surface recrystallization), see e.g. the cooling curves for both coverages in Fig. 1. It results from the fact that one measures the total electric signal between two macroscopic contacts which include many effects like the bulk Si conductivity, Si surface conductivity, the signal from $(6 \times 6) \mathrm{Au}$ superstructure and from deposited films. Of course, these components are not independent of each other and some interference effect always appear. On the other hand, the surface crystalline order at RT is stable and the temperature behaviour of the cooling curve, $G_{\text {cool }}$, corresponds mainly to under-surface effects (bulk semiconductor, phonons, and others). Thus we propose to analyze the normalized difference between the heating and cooling conductance curves, $\left(G_{\text {heat }}-G_{\text {cool }}\right) / G_{R T}$. The corresponding curves for both coverages, 0.65 ML and 4.2 ML are shown in Fig. 3, broken and solid curves, respectively. Now one can see that the conductance difference tends to near-zero value with increase of temperature which corresponds to stable crystalline order at the surface (for ultrathin $\mathrm{Pb}$ thickness it appears at about $175 \mathrm{~K}$ ). For higher temperatures $\left(G_{\text {heat }}-G_{\text {cool }}\right) / G_{R T}$ remains almost constant. Note that the conductance difference curve for the submonolayer coverage $0.65 \mathrm{ML}$ decreases at low temperature (up to $T=120 \mathrm{~K}$, Fig. 3, broken curve). It could be a consequence of very similar temperature dependence of the conductance for bare $\mathrm{Si}(111)-6 \times 6 \mathrm{Au}$ surface and $\mathrm{Si}(111)-6 \times 6 \mathrm{Au}$ with 0.65 ML-thick $\mathrm{Pb}$ film - both conductance curves strongly decreases below $120 \mathrm{~K}$, cf. also [29], leading to a local minimum in $\left(G_{\text {heat }}-G_{\text {cool }}\right) / G_{R T}$ for $T=120 \mathrm{~K}$. 


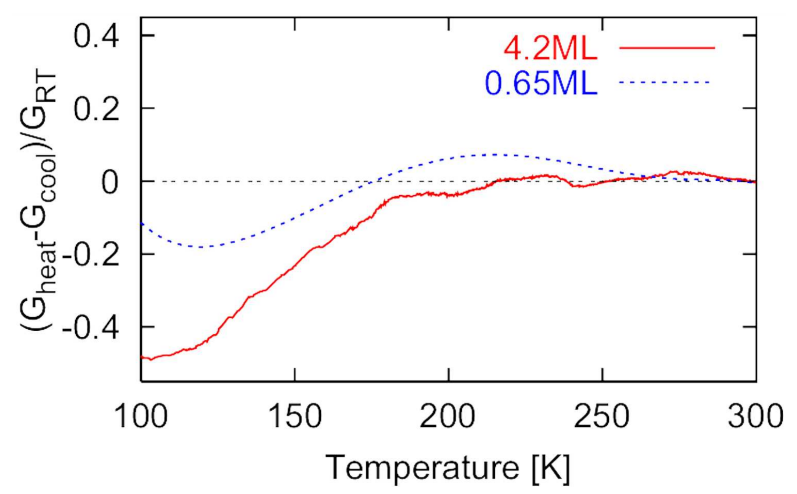

Fig. 3. Temperature dependence of the relative difference of the conductance obtained from the experimental data shown in Fig. $1,\left(G_{\text {heat }}-G_{\text {cool }}\right) / G_{R T}$, for $0.65 \mathrm{ML}$ of $\mathrm{Pb}$ (broken curve) and 4.2 ML of $\mathrm{Pb}$ (solid line) deposited on $\mathrm{Si}(111)-6 \times 6 \mathrm{Au}$ surface at $90 \mathrm{~K}$.

\section{Theoretical description}

The system under consideration consists of a thin film of $\mathrm{Pb}$ atoms deposited at low temperature onto the modified silicon substrate, $\mathrm{Si}(111)-6 \times 6 \mathrm{Au}$. The annealing process does not change the average thickness of the $\mathrm{Pb}$ film, however, deposited atoms move at the surface leading to the surface recrystallization. To describe our experimental results qualitatively one can use the TrivediAshcroft theory [36] which includes bulk impurity scattering and the surface roughness scattering. Within this theory the conductance of the system reads

$$
\sigma=\frac{e^{2} k_{\mathrm{F}}}{\hbar \pi} \frac{1}{\kappa} \sum_{m=1}^{n_{c}} \frac{1-\frac{m^{2}}{\kappa^{2}}}{\frac{2 n_{c}+1}{k_{\mathrm{F}} l_{0} \kappa}+\left(\frac{\delta d}{d}\right)^{2} \frac{s\left(n_{c}\right) m^{2}}{3 \kappa}},
$$

where $\kappa=\frac{k_{\mathrm{F}} d}{\pi}, k_{\mathrm{F}}$ is the Fermi wave vector, $l_{0}$ is the effective electron mean free path, $n_{c}=\operatorname{Int}(\kappa)$ and $s\left(n_{c}\right)=\left(2 n_{c}+1\right)\left(n_{c}+1\right) n_{c} / 3 \kappa^{3}$. Note that $\delta d$ function is the root-mean-square deviation of the mean film thickness, $d$, and can be obtained e.g. from the RHEED intensity oscillations during deposition of atoms at the substrate $[5,44,45]$. For the perfect growth of atoms (layer-by-layer mode) the roughness function can be expressed analytically in the parabolic form or for non-ideal growth it can be approximated by higher-order polynomials. In our case the sample is annealed from LNT up to RT (the thickness remains unchanged) and the roughness function, $\delta d$, changes with temperature. This function is responsible for the reconstruction of deposited atoms and formation of flat $\mathrm{Pb}$ areas at the surface (and is correlated with the STM topography images e.g. [33, 35, 37, 38]). At low temperature deposited atoms are placed randomly at the surface and their positions change with temperature. For submonolayer coverage, at higher temperature, $\mathrm{Pb}$ atoms form small 1 ML-thick clusters (composed of a few atoms $[37,38])$ thus in this case $(\delta d)^{2}$ decreases with temperature and can be expressed by a parabolic function, Fig. 4 (upper part, broken curve). For somewhat larger coverages, just after deposition, $\mathrm{Pb}$ atoms form irregular structures (see the left inset in Fig. 4) and, as the temperature increases, the surface becomes flatter and flatter with even-ML steps (see the right inset) [35]. In this case we express the roughness function in the form of 3rd order polynomial, Fig. 4 (upper part, solid curve). Note that we always work in the regime of $\left(\frac{\delta d}{d}\right)^{2}<1$, cf. also [41]. In our calculations we assume that the $\operatorname{Si}(111)-6 \times 6 \mathrm{Au}$ surface stands for an effective layer for electrons and thus the effective thickness of the sample, $d$, is $(0.65+1) \mathrm{ML}$ and $(4.2+1) \mathrm{ML}$, respectively. The thickness of $\mathrm{Pb}$ films is expressed in units of $1 \mathrm{ML}$ of $\mathrm{Pb}$, which equals about $0.42 \mathrm{~nm}[37,38]$.

In Fig. 4, bottom part, we show the relative changes of the normalized conductance, $\left(G_{\text {heat }}-G_{R T}\right) / G_{R T}$, during the annealing process (as a function of the temperature) for both considered thicknesses $0.65 \mathrm{ML}$, broken curve, and 4.2 ML, solid curve. The corresponding roughness functions are depicted in the upper part. In the calculations we assume that the average mean free path for

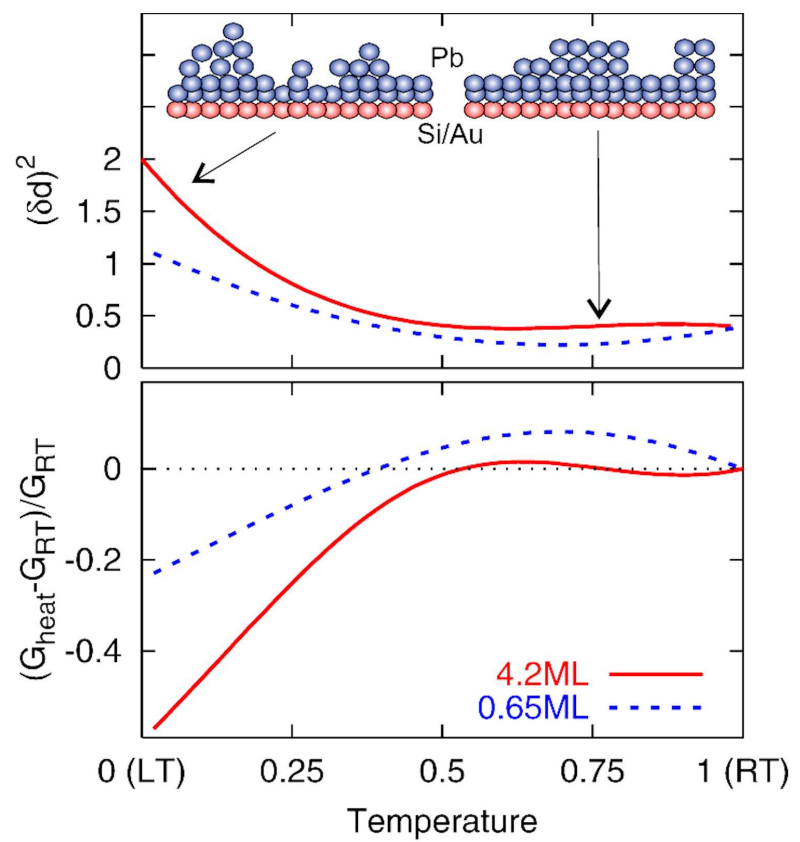

Fig. 4. The roughness function, $(\delta d)^{2}$, (upper part) and normalized relative conductance, $\left(G_{\text {heat }}-G_{R T}\right) / G_{R T}$ (bottom part) obtained according to the Trivedi-Ashcroft theory for different thickness of the $\mathrm{Pb}$ film: $0.65 \mathrm{ML}$ and $4.2 \mathrm{ML}$, broken and solid lines, respectively. The parameters used in the calculations are: $d=1.65, l_{0}=7$ for $0.65 \mathrm{ML}$ of $\mathrm{Pb}$ and $d=5.2, l_{0}=40$ for $4.2 \mathrm{ML}$ of $\mathrm{Pb}, k_{\mathrm{F}}=\pi$. The insets in the upper part show schematic distributions of $\mathrm{Pb}$ atoms after deposition at low temperature (left inset) and after annealing at room temperature (right inset).

electrons is limited mainly by the dimension of $6 \times 6 \mathrm{Au}$ cell for the submonolayer coverage (for $0.65 \mathrm{ML}$ ) and by the bulk mean free path for the case of $4.2 \mathrm{ML}$. As one can see, the curves increase with temperature and cross the zero value which is a hallmark of $\mathrm{Pb}$ recrystallization at the surface. The obtained results are in good qualitative agreement with the experimental data, Fig. 3. 


\section{Conclusions}

The electrical conductance of ultrathin $\mathrm{Pb}$ films on $\mathrm{Si}(111)-6 \times 6 \mathrm{Au}$ substrate was measured experimentally as a function of temperature using the four-point probe method in UHV condition. $\mathrm{Pb}$ atoms were deposited at LNT and then the conductance of the sample was measured during the heating (up to the room temperature) and cooling down cycle. The structure of the substrate with deposited films was monitored by the RHEED system.

We have found that the conductance increases after the annealing process as in this case the crystalline order of the surface improves. The conductance behaviour during the first heating and cooling run shows irreversible changes. It allows one to determine specific annealing temperature of the sample at which the conductance changes become temperature reversible. The most probable interpretation of this temperature is the surface recrystallization of $\mathrm{Pb}$ films and formation of $\mathrm{Pb}$ flat steps or island, which is supported by the STM topography images [35]. For $\mathrm{Pb}$ atoms at $\mathrm{Si}(111)-6 \times 6 \mathrm{Au}$ surface this temperature equals about $175 \mathrm{~K}$. Moreover, we have shown that $4 \mathrm{ML}$ of $\mathrm{Pb}$ at $\mathrm{Si}(111)-6 \times 6 \mathrm{Au}$ surface ensures metallic bulk-like behaviour of the sample conductance (the temperature coefficient of resistivity equals $0.00361 / \mathrm{K})$ while for other surface reconstructions much thicker $\mathrm{Pb}$ films are required.

We have also analyzed theoretically the conductance of thin films using the modified Trivedi-Ashcroft theory for fixed thickness of the sample. It was shown that the relative conductance depends mainly on the surface roughness which changes during the annealing process. Satisfactory qualitative agreement with the experimental data was obtained.

\section{Acknowledgments}

This work was partially supported by National Science Centre, Poland, under Grant No. 2014/13/ B/ST5/04442.

\section{References}

[1] M.K. Weldon, K.T. Queeney, J. Eng, K. Raghavachari, Y.J. Chabal, Surf. Sci. 500, 859 (2002).

[2] Y. Jia, B. Wu, C. Li, T.L. Einstein, H.H. Weitering, Z. Zhang, Phys. Rev. Lett. 105, 066101 (2010).

[3] M. Bouhassoune, B. Zimmermann, P. Mavropoulos, D. Wortmann, P. Dederichs, S. Blugel, S. Lounis, $\mathrm{Na}$ ture Commun. 5, 5558 (2014).

[4] M. Jałochowski, E. Bauer, Phys. Rev. B 38, 5272 (1988).

[5] M. Jałochowski, E. Bauer, H. Knoppe, G. Lilienkamp, Phys. Rev. B 45, 13607 (1992).

[6] M. Jałochowski, M. Hoffmann, E. Bauer, Phys. Rev. Lett. 76, 4227 (1996)10.1103/PhysRevLett.76.4227 Phys. Rev. B 51, 7231 (1995).

[7] I. Vilfan, M. Henzler, O. Pfennigstorf, H. Pfnur, Phys. Rev. B 66, 241306 (2002).
[8] K.v. Klitzing, G. Dorda, M. Pepper, Phys. Rev. Lett. 45, 494 (1980).

[9] I. Drozdov, A. Alexandradinata, S. Jeon, S. NadjPerge, H. Ji, R. Cava, B. Bernevig, A. Yazdani, $\mathrm{Na}$ ture Phys. 10, 664 (2014).

[10] Y. Xia, D. Qian, D. Sieh, L. Wray, A. Pal, H. Lin, A. Bansil, D. Grauer, Y. Hor, R. Cava, M. Hasan, Nature Phys. 5, 398 (2009).

[11] Z. Korczak, T. Kwapiński, Surf. Sci. 601, 3324 (2007).

[12] O. Pfennigstorf, A. Petkova, Z. Kallassy, M. Henzler, Eur. Phys. J. B 30, 111 (2002).

[13] Z. Korczak, T. Kwapiński, Surf. Sci. 600, 1650 (2006).

[14] S. Hasegawa, X. Tong, S. Takeda, N. Sato, T. Nagao, Prog. Surf. Sci. 60, 89 (1999).

[15] T. Kwapiński, J. Phys. Condens. Matter 19, 176218 (2007)10.1088/0953-8984/19/17/176218 J. Phys. Condens. Matter 22, 295303 (2010).

[16] S.M. Binz, M. Hupalo, M.C. Tringides, Phys. Rev. B 78, 193407 (2008).

[17] M. Jałochowski, E. Bauer, Surf. Sci. 480, 109 (2001).

[18] W. Su, S. Chang, W. Jian, C. Chang, L. Chen, T. Tsong, Phys. Rev. Lett. 86, 5116 (2001).

[19] Z. Korczak, T. Kwapiński, Surf. Sci. 604, 54 (2010).

[20] T. Ala-Nissila, R. Ferrando, S.C. Ying, Adv. Phys. 51, 949 (2002).

[21] T.-L. Chan, C.Z. Wang, M. Hupalo, M.C. Tringides, K.M. Ho, Phys. Rev. Lett. 96, 226102 (2006).

[22] K.R. Roos, M.C. Tringides, Phys. Rev. Lett. $\mathbf{8 5}$ 1480 (2000)

[23] K. Man, M. Tringides, M. Loy, M. Altman, Phys. Rev. Lett. 110, 036104 (2013).

[24] P. Nita, M. Jałochowski, M. Krawiec, A. Stepniak, Phys. Rev. Lett. 107, 026101 (2011).

[25] C. Tegenkamp, Z. Kallassy, H. Pfnur, H.-L. Gunter, V. Zielasek, M. Henzler, Phys. Rev. Lett. 95, 176804 (2005).

[26] S. Hasegawa, F. Grez, Surf. Sci. 500, 84 (2002).

[27] S. Hasegawa, J. Phys. Condens. Matter. 12, R463 (2000).

[28] T. Uetake, T. Hirahara, Y. Ueda, N. Nagamura, R. Hobara, S. Hasegawa, Phys. Rev. B 86, 035325 (2012).

[29] S. Yamazaki, I. Matsuda, H. Okino, H. Morikawa, S. Hasegawa, e-J. Surf. Sci. Nanotech. 3, 497 (2005).

[30] S. Yamazaki, Y. Hosomura, I. Matsuda, R. Hobara, T. Eguchi, Y. Hasegawa, S. Hasegawa, Phys. Rev. Lett. 106, 116802 (2011).

[31] S. Hatta, T. Noma, H. Okuyama, T. Aruga, Phys. Rev. B 90, 245407 (2014).

[32] O. Pfennigstorf, K. Lang, H. Gunter, M. Henzler, Appl. Surf. Sci. 162, 537 (2000).

[33] M. Jałochowski, Progr. Surf. Sci. 74, 97 (2003).

[34] T. Schmidt, E. Bauer, Phys. Rev. B 62, 15815 (2000).

[35] M. Jałochowski, K. Palotas, M. Krawiec, Phys. Rev. B 93, 035437 (2016). 
[36] N. Trivedi, N. Ashcroft, Phys. Rev. B 38, 12298 (1988).

[37] M. Jałochowski, M. Stóżak, R. Zdyb, Phys. Rev. B 66, 205417 (2002).

[38] M. Kisiel, M. Jałochowski, R. Zdyb, Phys. Lett. A 357, 141 (2006).

[39] Y.-F. Zhang, J.-F. Jia, Z. Tang, T.-Z. Han, Xu-Cun Ma, Qi-Kun Xue, Surf. Sci. 596, L331 (2005).

[40] V. Yeh, M. Hupalo, E.H. Conrad, M.C. Tringides, Surf. Sci. 551, 23 (2004).

[41] P. Czoschke, H. Hong, L. Basile, T.-C. Chiang, Phys. Rev. B 72, 075402 (2005).
[42] L. Floreano, D. Cvetko, F. Bruno, G. Bavdek, A. Cossaro, R. Gotter, A. Verdini, A. Morgante, Progr. Surf. Sci. 72, 135 (2003).

[43] R.A. Serway, J.R. Gordon, Principles of Physics (2nd ed.), Saunders College Pub., London 1998.

[44] P.I. Cohen, G.S. Petrich, P.R. Pukite, G.J. Whaley, S. Arrott, Surf. Sci. 216, 222 (1989).

[45] M. Horn, V. Gotter, M. Henzler, in: Reflection HighEnergy Electron Diffraction and Reflection Electron Imaging of Surface, Eds. P.K. Larsen, P.J. Dobson, Plenum, New York 1988, p. 463. 